\title{
Effects of Neurostimulation on Poststroke Dysphagia: A Synthesis of Current Evidence From Randomized Controlled Trials
}

\section{Ivy Cheng, PhD $\odot$; Ayodele Sasegbon, MBChB; Shaheen Hamdy, PhD}

\begin{abstract}
Objectives: To evaluate the effects of neurostimulation, including repetitive transcranial magnetic stimulation (rTMS), transcranial direct current stimulation (tDCS) and pharyngeal electrical stimulation (PES), for poststroke dysphagia based on evidence from randomized controlled trials (RCTs).

Materials and Methods: Electronic databases were systematically searched between January 1985 and June 2020 and studies were included based on prespecified selection criteria. The quality of studies was evaluated and data were extracted and synthesized by two independent reviewers. The primary outcome measure was change in (any) relevant clinical swallowingrelated characteristic. Subgroup analysis were conducted based on follow-up period and stimulation parameters.

Results: Data from 852 stroke patients were collected from 26 RCTs studies. Active neurostimulation treatments demonstrated a significant and moderate effect size compared to control treatment $(0.69[95 \% \mathrm{Cl}=0.50,0.89] ; p<0.001)$. The effect size of rTMS was the largest $(0.73[95 \% \mathrm{Cl}=0.49,0.98] ; p<0.001)$, followed by PES $(0.68[95 \% \mathrm{Cl}=0.22,1.14] ; p=0.004)$ and tDCS $(0.65$ $[95 \% \mathrm{Cl}=0.25,1.04] ; p=0.001)$. All treatments showed comparable effect sizes within the first two weeks. Between three weeks and two months, tDCS demonstrated the largest effects $(1.02[95 \% \mathrm{Cl}=0.45,1.59] ; p<0.001)$ among the three treatments. No significant treatment effects were reported beyond three months. The combined effect size was large when applied in acute ( $<14$ days) stroke $(0.8[95 \% \mathrm{Cl}=0.34,1.26] ; p<0.001)$. For noninvasive brain stimulation (NIBS), bihemispheric stimulation demonstrated the strongest effect size $(0.93[95 \% \mathrm{Cl}=0.53,1.33] ; p<0.001)$. In contrast, unilateral rTMS using ipsilesional highfrequency stimulation had a combined effect size of $0.83(95 \% \mathrm{Cl}=0.14,1.52 ; p=0.02)$. For tDCS, a significant effect size was found only with anodal stimulation applied over the contralesional hemisphere $(1.04[95 \% \mathrm{Cl}=0.54,1.53] ; p<0.001)$.

Conclusions: The results show that neurostimulation can benefit patients with poststroke dysphagia. The treatment effects were the strongest in acute stroke patients and within the first two months of application. For NIBS, bihemispheric stimulation appeared to be most effective. The most beneficial hemisphere for unilateral stimulation differed between rTMS and tDCS. These findings provide a platform for future studies and clinical practice.
\end{abstract}

Keywords: Dysphagia, dysphagia treatment, meta-analysis, neurostimulation, rehabilitation, stroke, systematic review

Conflict of Interest: Shaheen Hamdy is chief scientific officer, a shareholder, and a board member of Phagenesis Ltd., a company that focuses on dysphagia therapies, specifically pharyngeal electrical stimulation. Shaheen Hamdy has also received research funding from MRC, Wellcome Trust, Stroke Association, and NIHR to explore brain stimulation strategies to treat poststroke dysphagia. All other authors declare no conflicts of interest.

\section{INTRODUCTION}

Swallowing disorders (dysphagia) are a common complication following stroke, with reported incidence ranging from $37 \%$ to $78 \%$ (1). The physical and psychosocial consequences of dysphagia are devastating. It is associated with malnutrition, dehydration, aspiration pneumonia, prolonged hospital stays, and increased mortality (1-3). Patients often experience anxiety, discomfort, and embarrassment during mealtime, leading to social withdrawal and poor quality of life (4). Dysphagia may resolve spontaneously during the first few weeks of stroke, but more than $50 \%$ of patients have persistent dysphagia at hospital discharge $(2,5)$. The healthcare cost associated with poststroke dysphagia is high and the persistence of dysphagia leads to long-term financial and social burdens for stroke patients $(6,7)$. Management of poststroke dysphagia includes compensatory strategies such as modification
Address correspondence to: Shaheen Hamdy, PhD, Centre for Gastrointestinal Sciences, Clinical Sciences Building, Salford Royal Foundation Trust, Eccles Old Road, Salford M6 8HD, UK. Email: shaheen.hamdy@manchester.ac.uk

Centre for Gastrointestinal Sciences, Division of Diabetes, Endocrinology and Gastroenterology, School of Medical Sciences, Faculty of Biology, Medicine and Health, University of Manchester, Manchester, UK

For more information on author guidelines, an explanation of our peer review process, and conflict of interest informed consent policies, please go to http:// www.wiley.com/WileyCDA/Section/id-301854.html

Source(s) of financial support: None.

This is an open access article under the terms of the Creative Commons Attribution-NonCommercial License, which permits use, distribution and reproduction in any medium, provided the original work is properly cited and is not used for commercial purposes. 
of diet consistencies, eating postures and feeding methods, rehabilitative exercises of swallowing musculatures to establish strength and coordination, acupuncture, and physical sensory stimulation such as tactile or thermal stimulation (8). However, the efficacy of these treatments remains controversial (8).

The lack of effective treatment has led researchers to explore alternative options to promote recovery through enhancing neural plasticity. Swallowing is regulated by the central nervous system involving structures from cerebral cortex to cranial nerves (9). Early studies on anesthetised and awake animals demonstrated bi-hemispheric cortical control of swallowing musculature $(10,11)$. In humans, Hamdy and colleagues (12) found that swallowing musculature (mylohyoid, pharyngeal, and esophageal muscles) are somatotopically represented in the human motor and premotor cortex, with interhemispheric asymmetry. Lesion studies in stroke patients have shown that damage to these cortical structures results in dysphagia (13). Importantly, recovery from dysphagia following unilateral hemispheric stroke is associated with increased cortical representation of the intact hemisphere (14), suggesting that the compensatory reorganization (neural plasticity) within this neural network is critical for recovery. Such neural plasticity can be promoted by noninvasive brain stimulation (NIBS), which has received growing attention in the recent years (15). Repetitive transcranial magnetic stimulation (rTMS) and transcranial direct current stimulation (tDCS) are two forms of NIBS approaches that modulates brain activity and induce long-lasting changes in synaptic plasticity (16). RTMS uses electromagnetic induction to depolarize postsynaptic connections (17), whereas tDCS uses direct electrical current to shift the polarity of nerve cells (18). A number of systematic reviews have shown that both techniques can improve swallowing functions following stroke, although most reviews have focused on acute and subacute stroke patients (19-23).

Apart from centrally or cortically applied neurostimulation, stimulation of the peripheral neural pathway may have therapeutic potential for poststroke dysphagia rehabilitation. Pharyngeal electrical stimulation (PES), which refers to direct stimulation of the pharyngeal mucosa through intraluminal catheter, is an example of such stimulation (24). Early studies in healthy volunteers showed that PES at $5 \mathrm{~Hz}$ and $75 \%$ of maximum tolerated intensity for 10 min can increase cortical excitability of the pharyngeal motor cortex for about $1 \mathrm{~h}$ (25). These findings suggested that peripheral neurostimulation could achieve centralized effects in driving plasticity of the nervous system. Several further studies have shown therapeutic potentials of PES in rehabilitation of poststroke dysphagia (25-28).

Despite the growing interests in neurostimulation as treatments for poststroke dysphagia, existing studies have small sample sizes which limits the ability to draw definitive conclusions on the effectiveness of such interventions. Therefore, we aimed to systematically review and synthesize the evidence from randomized controlled trials (RCTs) of neurostimulation for poststroke dysphagia. In this review, we focused on rTMS, tDCS and PES because these treatments have demonstrated the ability to modulate neural plasticity from previous studies (29). Subgroup analysis was performed to analyze the treatment effects based on the time of follow-ups, chronicity of stroke and stimulation paradigms to provide insights into future best practice for neurostimulation.

\section{MATERIALS AND METHODS}

This systematic review and meta-analysis followed the Preferred Reporting Items for Systematic Reviews and Meta-Analyses (PRISMA) guidelines. Two reviewers performed the search for studies, data extraction and risk of bias assessment independently. Data synthesis was carried out by one reviewer and verified by a second reviewer. Disagreements were resolved by consensus among all authors.

\section{Study Identification and Search Method}

We searched the following electronic databases from January 1985 to June 2020: MEDLINE (via PubMed), EMBASE (via Ovid), and Cochrane Library. Citations from identified papers were tracked and systematic reviews were searched manually for relevant references. The terms used for searches included: dysphagia, swallowing disorders, deglutition disorders, swallowing, deglutition, poststroke, stroke, cerebrovascular accident, infarction, neurostimulation, cortical stimulation, brain stimulation, transcranial magnetic stimulation (TMS), transcranial direct current stimulation (tDCS), and pharyngeal electrical stimulation (PES).

\section{Eligibility Criteria}

We included all randomized controlled trial studies that compared neurostimulation ( $r T M S$, tDCS, and PES) with sham stimulation or other interventions for post-stroke dysphagia (predominantly standard care). Case studies, open-label studies, animal studies, observational studies, quasi-experimental studies, studies on healthy volunteers and studies that did not include original data were excluded. Non-English studies were excluded.

\section{Participants}

Studies with adult participants who were diagnosed with poststroke dysphagia regardless of the time of onset or type of stroke (ischemic, haemorrhagic or brainstem infarction) were included. Studies with patients whose dysphagia was caused by other aetiologies, for example, traumatic brain injury, neurodegenerative diseases or motor neuron diseases, were excluded.

\section{Interventions}

We included studies that compared neurostimulation (rTMS, tDCS, and PES) with placebo stimulation or head-to-head comparisons of different types of neurostimulation for post-stroke dysphagia. Conventional dysphagia therapy was accepted as a comparator. Trials with multiple interventions (e.g., co-administration of neurostimulation and conventional swallowing therapy) were eligible if the study groups only differed by the use of the target neurostimulation of interest.

\section{Outcomes}

Study outcomes related to swallowing, which included swallowing physiology measurement, clinical swallowing function ratings, functional dysphagia symptom scales or health outcomes related to swallowing or pharyngeal functions were included for comparisons.

\section{Data Extraction}

The data extracted included: demographic information of participants (age, gender, and stroke characteristics), stimulation protocol (intensity, location, and duration), outcomes (mean [standard deviation] or median [interquartile range]) and sample sizes. For studies with multiple outcome measures, the most relevant primary swallowing-related outcome was used. If data were not provided, we attempted to contact the corresponding authors. If data were presented in figures and raw data were not obtainable from the authors, an online plot digitalizer program (WebPlotDigitizer 4.3; 
https://apps.automeris.io/wpd/; USA) was used to extract graphic data. If only partial data were reported and we were unable to contact the corresponding authors, imputation was used to estimate the results (30). If data were not obtainable despite these attempts, the study was excluded from the review.

\section{Risk of Bias Assessment}

Seven domains of risk of bias of RCTs were evaluated using the Cochrane Collaboration's tool for assessing risk of bias (31). These

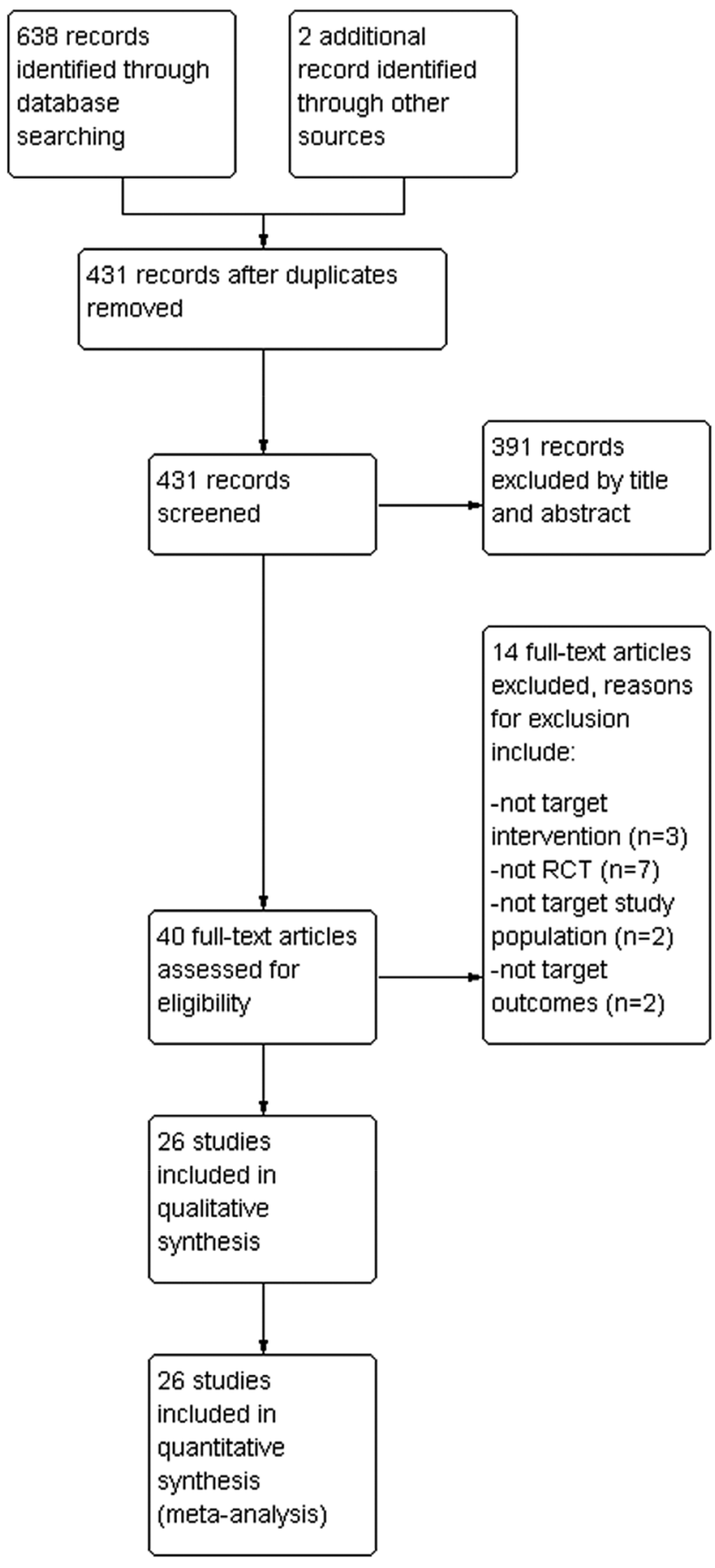

Figure 1. Flow diagram for study identification and inclusion. domains included random sequence generation, allocation concealment, blinding of participants and personnel, blinding of outcome assessment, incomplete data, selective reporting and other sources of bias. Two reviewers rated the risk of bias of the included studies independently, followed by discussions among all authors to resolve any disagreements on their judgments.

\section{Statistical Analysis}

All statistical analyses were performed by Review Manager 5.4 software program (RevMan; Cochrane Collaboration, Oxford, UK). The treatment effects were determined by comparing the treatment outcomes against that of the comparators. Studies with multiple interventions groups were analyzed separately for each experimental-control comparison, with the sample size of the "shared" control group being split equally for each comparison (31). Data extracted for treatment effect calculation included group sizes, group mean differences and pooled standard deviations. For studies that reported median and interquartile range, the mean and standard deviations were estimated using methods previously described $(32,33)$. For outcome measures that increases with disease severity, the mean values were multiplied by -1 . Pooled standard deviation was calculated using the following formula (34):

$\mathrm{SD}_{\text {pooled }}=\sqrt{\frac{\left(n_{\text {pre }}-1\right) \mathrm{SD}_{\text {pre }}^{2}+\left(n_{\text {post }}-1\right) \mathrm{SD}_{\text {post }}^{2}}{n_{\text {pre }}+n_{\text {post }}-2}}$

Treatment effects for continuous outcomes were analyzed as standardized mean difference (SMD) with 95\% confidence intervals. For dichotomous outcomes, the odds ratios (OR) were first calculated and then converted to standardized mean difference using the formula below, again as previously described (31):

$\mathrm{SMD}=\frac{\sqrt{3}}{\pi} \operatorname{lnOR}$

The dichotomous outcomes were then combined with continuous outcomes for comparisons using generic inverse-variance method from the RevMan program (31).

A weighted average of SMD across studies was computed using random effects model analysis. The significance level was set at $p<0.05$ and the effect sizes were presented as SMD (95\% confidence interval; $\mathrm{Cl}$ ). For the interpretation of effect sizes, SMD of 0.2 represented a small effect, 0.5 a moderate effect, and 0.8 a large effect (34). Heterogeneity was assessed with Cochrane's $Q$ statistic and $l^{2}$ test in which heterogeneity was considered substantial with $p<0.05$ and $l^{2}$ higher than 50\%. Subgroup analysis was conducted based on the follow-up period to evaluate the treatment effects over time and based on chronicity of stroke to evaluate the effects of timing of treatment (see definitions in Results section). Due to the diversity of rTMS and tDCS paradigms, subgroup analysis was performed only for the stimulated hemisphere and rTMS stimulation frequency.

\section{RESULTS}

Figure 1 showed the flow diagram of study identification. A total of 638 studies were identified from three electronic databases and two from other sources, of which 431 studies were 
Table 1. Characteristics of Included Studies.

\begin{tabular}{|c|c|c|c|c|c|c|c|c|}
\hline Study & Stimulation protocol & Stimulation target & Comparison & Sample size & $\begin{array}{l}\text { Age (years) } \\
\text { Mean (SD) }\end{array}$ & $\begin{array}{l}\text { Poststroke onset } \\
\text { duration (days) } \\
\text { Mean (SD) }\end{array}$ & Follow-up schedule & $\begin{array}{l}\text { Primary } \\
\text { outcome }\end{array}$ \\
\hline \multicolumn{9}{|l|}{ rTMS } \\
\hline (35) & $\begin{array}{l}3 \mathrm{~Hz} / 120 \% \text { hand } \mathrm{rMT} / 300 \text { pulses } \\
(10 \mathrm{~min}) ; \text { five days }\end{array}$ & iEMC & Active vs. sham & $14 / 12$ & $57.3(12.5)$ & $5-10$ & $\begin{array}{l}\text { Immediate } \\
\text { One month } \\
\text { Two months }\end{array}$ & DD \\
\hline (36) & $\begin{array}{l}3 \mathrm{~Hz} / 130 \% \text { hand } \mathrm{rMT} / 300 \text { pulses } \\
\text { (each hemisphere) }(10 \mathrm{~min}) \\
\text { five days }\end{array}$ & Both EMC & Active vs. sham & $\begin{array}{l}\text { Brainstem: } 5 / 6 \\
\text { LMI: } 6 / 5\end{array}$ & $57.3(12.6)$ & $32.2(16.8)$ & $\begin{array}{l}\text { Immediate } \\
\text { One month } \\
\text { Two months }\end{array}$ & $\mathrm{DD}$ \\
\hline (37) & $\begin{array}{l}5 \mathrm{~Hz} / 100 \% \text { mylohyoid } \mathrm{rMT} / 1000 \\
\text { pulses } \\
(20 \mathrm{~min}) ; 1 \mathrm{~Hz} / 100 \% \text { mylohyoid } \\
\text { rMT/1200 pulses } \\
(20 \mathrm{~min}) ; \text { ten days }\end{array}$ & $\begin{array}{l}5 \mathrm{~Hz}: \text { iMMC } \\
1 \mathrm{~Hz}: \mathrm{CMMC} \\
\text { Sham: iMMC }\end{array}$ & $\begin{array}{c}5 \mathrm{~Hz}+\mathrm{CDT} \text { vs. } \\
1 \mathrm{~Hz}+\mathrm{CDT} \text { vs. } \\
\text { sham + CDT }\end{array}$ & $10 / 10 / 10$ & $68.1(10.9)$ & $29.9(23.9)$ & Immediate & PAS \\
\hline (38) & $\begin{array}{l}5 \mathrm{~Hz} / 90 \% \text { hand } \mathrm{rMT} / 500 \text { pulses } \\
(10 \mathrm{~min}) ; \text { ten days }\end{array}$ & CPMC & Active vs. sham & $9 / 9$ & $71.3(7.3)$ & $61.9(21.6)$ & $\begin{array}{l}\text { Immediate } \\
\text { two weeks }\end{array}$ & PAS \\
\hline (39) & $\begin{array}{l}5 \mathrm{~Hz} / 90 \% \text { hand } \mathrm{rMT} / 250 \text { pulses } \\
\text { ( 2 } \mathrm{min}) \text {; one day }\end{array}$ & CPMC & $\begin{array}{l}\text { Active vs. sham } \\
\text { (cross-over) }\end{array}$ & $6 / 6$ & $67.3(7.7)$ & $212.1(164.5)$ & Immediate & PAS \\
\hline (40) & $\begin{array}{l}1 \mathrm{~Hz} / 100 \% \text { mylohyoid } \mathrm{rMT} / 1200 \\
\text { pulses (20 min); ten days }\end{array}$ & CPMC & $\begin{array}{l}\text { CDT + rTMS vs. CDT } \\
\text { + NMES vs. CDT }\end{array}$ & $14 / 18 / 15$ & $\begin{array}{l}63.1 \\
(12.4)\end{array}$ & $32.4(12.5)$ & $\begin{array}{l}\text { Immediate } \\
\text { two weeks }\end{array}$ & PAS \\
\hline (41) & $\begin{array}{l}3 \mathrm{~Hz} / 90 \% \text { mylohyoid rMT/1200 } \\
\text { pulses ( 13 min); } 1 \mathrm{~Hz} / 100 \% \\
\text { mylohyoid rMT/1200 pulses } \\
\text { ( 21 min); five days }\end{array}$ & $\begin{array}{l}3 \mathrm{~Hz}: \mathrm{iMMC} \\
1 \mathrm{~Hz}: \mathrm{CMMC} \\
\text { Sham: } \mathrm{CMMC}\end{array}$ & $\begin{array}{l}\text { Ipsilesional } 3 \mathrm{~Hz} \text { vs. } \\
\text { contralesional } 1 \mathrm{~Hz} \\
\text { vs. sham }\end{array}$ & $13 / 13 / 12$ & $58.3(2.8)$ & $12.6(15.5)$ & $\begin{array}{l}\text { Immediate } \\
\text { One month } \\
\text { Two months } \\
\text { Three months }\end{array}$ & SSA \\
\hline (42) & $\begin{array}{l}10 \mathrm{~Hz} / 90 \% \text { mylohyoid rMT/500 } \\
\text { pulses (10 min each } \\
\text { hemisphere); ten days }\end{array}$ & $\begin{array}{l}\text { Bihemispheric: MMC } \\
\text { Unilateral: iMMC } \\
\text { Sham: bihemispheric }\end{array}$ & $\begin{array}{l}\text { Bihemispheric vs. } \\
\text { ipsilesional } 10 \mathrm{~Hz} \text { vs. } \\
\text { sham }\end{array}$ & $11 / 11 / 11$ & $65.9(12.4)$ & $35(33.6)$ & $\begin{array}{l}\text { Immediate } \\
\text { two weeks }\end{array}$ & PAS \\
\hline (43) & $\begin{array}{l}5 \mathrm{~Hz} / 90 \% \text { tongue rMT/3000 pulses } \\
\quad(\sim 18 \mathrm{~min}) ; \text { ten days }\end{array}$ & ITMC & Active vs. sham & $11 / 4$ & $64.6(7.9)$ & $1251(618)$ & $\begin{array}{l}\text { Two months } \\
\text { Six months } \\
12 \text { months }\end{array}$ & $\mathrm{PTT}$ \\
\hline (44) & $\begin{array}{l}1 \mathrm{~Hz} / 120 \% \text { mylohyoid } \mathrm{rMT} / 1200 \\
\text { pulse (20 min); five days }\end{array}$ & CMMC & $\begin{array}{l}\text { CDT + rTMS vs. rTMS vs. } \\
\text { CDT }\end{array}$ & $6 / 6 / 6$ & $60.7(14.8)$ & $96(54)$ & $\begin{array}{l}\text { Immediate } \\
\text { Two weeks } \\
\text { Four weeks } \\
\text { Five weeks }\end{array}$ & MASA \\
\hline (45) & $\begin{array}{l}1 \mathrm{~Hz} / 90 \% \text { mylohyoid MT/1200 } \\
\text { pulses (20 min); five days }\end{array}$ & $\mathrm{CMMC}$ & CDT + rTMS vs. CDT & $15 / 13$ & $68.5(12.1)$ & $103.8(45.1)$ & $\begin{array}{l}\text { Immediate } \\
\text { One month } \\
\text { Three months }\end{array}$ & PAS \\
\hline (46) & $\begin{array}{c}10 \mathrm{~Hz} / 110 \% \text { mylohyoid rMT/900 } \\
\text { pulses ( } 15 \mathrm{~min}) ; 1 \mathrm{~Hz} / 80 \% \\
\text { mylohyoid rMT/900 pulses } \\
(15 \mathrm{~min}) ; \text { ten days }\end{array}$ & $\begin{array}{l}\text { Bihemispheric: } 10 \mathrm{~Hz} \\
\quad \text { iMMC } \rightarrow 1 \mathrm{~Hz} \\
\quad \text { CMMC } \\
10 \mathrm{~Hz}: \text { iMMC } \\
1 \mathrm{~Hz}: \text { CMMC }\end{array}$ & $\begin{array}{l}\text { Bihemispheric vs. } \\
\text { ipsilesional } 10 \mathrm{~Hz} \text { vs. } \\
\text { contralesional } 1 \mathrm{~Hz} \\
\text { vs. sham } \\
\text { (all +NMES) }\end{array}$ & $16 / 16 / 16 / 16$ & $55.6(9.7)$ & $23.7(7.3)$ & $\begin{array}{l}\text { Immediate } \\
\text { One month }\end{array}$ & SSA \\
\hline (47) & $\begin{array}{l}5 \mathrm{~Hz} / 90 \% \text { hand } \mathrm{rMT} / 250 \text { pulses } \\
\text { ( 2 } \mathrm{min}) ; \text { one day }\end{array}$ & CPSC & $\begin{array}{l}\text { Active vs. sham (cross- } \\
\text { over) }\end{array}$ & $12 / 12 / 12$ & $70.0(8.6)$ & $493.1(672.4)$ & Immediate & PAS \\
\hline \multicolumn{9}{|c|}{ (2. } \\
\hline (48) & $2 \mathrm{~mA} / 30 \mathrm{~min}$; five days & ilsC & $\begin{array}{l}\text { Active + CDT vs. sham } \\
\quad+\text { CDT }\end{array}$ & $7 / 7$ & $71.6(21.4)$ & $3.7(1.8)$ & immediate & DOSS \\
\hline (49) & $1 \mathrm{~mA} / 20 \mathrm{~min}$; ten days & iPMC & $\begin{array}{l}\text { Active }+ \text { CDT vs. sham } \\
\quad+\text { CDT }\end{array}$ & $9 / 7$ & $71.0(10.8)$ & $25.9(10.2)$ & $\begin{array}{l}\text { Immediate } \\
\text { Three months }\end{array}$ & FDS \\
\hline (50) & $1 \mathrm{~mA} / 20 \mathrm{~min}$; ten days & iPMC & $\begin{array}{l}\text { Active }+ \text { CDT vs. sham } \\
\quad+\text { CDT }\end{array}$ & $10 / 10$ & $65.8(7.8)$ & $87.5(58.8)$ & $\begin{array}{l}\text { Immediate } \\
\text { One month }\end{array}$ & DOSS \\
\hline (51) & $1 \mathrm{~mA} / 20 \mathrm{~min}$; ten days & Bilateral PMC & $\begin{array}{l}\text { Active }+ \text { CDT vs. sham } \\
\quad+\text { CDT }\end{array}$ & $13 / 13$ & $64(10.5)$ & $357(141)$ & Immediate & DOSS \\
\hline (52) & $1 \mathrm{~mA} / 20 \mathrm{~min}$; four days & CPMC & $\begin{array}{l}\text { Active + CDT vs. sham } \\
\quad+\text { CDT }\end{array}$ & $29 / 30$ & $68.0(13.0)$ & $4.9(0.3)$ & Immediate & DSRS \\
\hline (53) & $2 \mathrm{~mA} / 30 \mathrm{~min} ;$ ten days & Bilateral PMC & $\begin{array}{l}\text { Active }+ \text { CDT vs. sham } \\
\quad+\text { CDT }\end{array}$ & $20 / 20$ & $64.9(13.4)$ & $<30$ & immediate & DOSS \\
\hline (54) & $1 \mathrm{~mA} / 20 \mathrm{~min} ; 20$ days & Bilateral EMC & $\begin{array}{l}\text { Active + balloon } \\
\text { dilation }+ \text { CDT vs. } \\
\text { sham + balloon } \\
\text { dilation + CDT }\end{array}$ & $14 / 14$ & $61.7(10.6)$ & $67.2(42.5)$ & Immediate & FDS \\
\hline (25) & $\begin{array}{l}5 \mathrm{~Hz} / 75 \% \text { tolerated threshold/ } \\
10 \mathrm{~min} \text {; one day }\end{array}$ & Pharynx & Active vs. sham & $10 / 6$ & $74.2(9.3)$ & $4(0.5)$ & Immediate & PAS \\
\hline (28) & $\begin{array}{l}5 \mathrm{~Hz} / 75 \% \text { tolerated threshold/ } \\
10 \mathrm{~min} \text {; three days }\end{array}$ & Pharynx & Active vs. sham & $16 / 12$ & $73(11)$ & $11-13$ days & Two weeks & DSR \\
\hline (39) & $\begin{array}{l}5 \mathrm{~Hz} / 75 \% \text { tolerated threshold/ } \\
10 \mathrm{~min} \text {; one day }\end{array}$ & Pharynx & $\begin{array}{l}\text { Active vs. sham } \\
\text { (cross-over) }\end{array}$ & $6 / 6$ & $60.3(16.8)$ & $623(429.8)$ & Immediate & PAS \\
\hline (27) & $\begin{array}{l}5 \mathrm{~Hz} / 75 \% \text { tolerated threshold/ } \\
10 \mathrm{~min} \text {; three days }\end{array}$ & Pharynx & Active vs. sham & $20 / 10$ & $64.2(14.4)$ & $25.8(12.1)$ & Immediate & $\begin{array}{l}\% \text { of } \\
\text { decannulation }\end{array}$ \\
\hline (55) & $\begin{array}{l}5 \mathrm{~Hz} / 75 \% \text { tolerated threshold/ } \\
10 \mathrm{~min} \text {; three days }\end{array}$ & Pharynx & Active vs. sham & $18 / 17$ & $69.9(14.6)$ & $13(9.3)$ & $\begin{array}{l}\text { Immediate } \\
\text { Three months }\end{array}$ & DSR \\
\hline (56) & $\begin{array}{l}5 \mathrm{~Hz} / 75 \% \text { tolerated threshold/ } \\
10 \mathrm{~min} \text {; three days }\end{array}$ & Pharynx & Active vs. sham & $70 / 50$ & $74.4(11.2)$ & $13.4(9.7)$ & $\begin{array}{l}\text { Immediate } \\
12 \text { weeks }\end{array}$ & PAS \\
\hline (26) & $\begin{array}{l}5 \mathrm{~Hz} / 75 \% \text { tolerated threshold/ } \\
10 \mathrm{~min} \text {; three days }\end{array}$ & Pharynx & Active vs. sham & $35 / 34$ & $64.2(11.9)$ & 19.-50.5 & Immediate & $\begin{array}{l}\% \text { of } \\
\text { decannulation }\end{array}$ \\
\hline (47) & $\begin{array}{l}5 \mathrm{~Hz} / 75 \% \text { tolerated threshold/ } \\
10 \mathrm{~min} \text {; one day }\end{array}$ & Pharynx & $\begin{array}{l}\text { Active vs. sham } \\
\text { (cross-over) }\end{array}$ & $12 / 12$ & $70.0(14.2)$ & $485.2(318.3)$ & Immediate & PAS \\
\hline
\end{tabular}

rMT: resting motor threshold; i: ipsilesional; c: contralesional; EMC: esophageal motor cortex; LMI: lateral medullary infarction; DD: degree of dysphagia; MMC: mylohyoid motor cortex; CDT: conventional dysphagia therapy; PAS: penetration aspiration scale; PMC: pharyngeal motor cortex; NMES: neuromuscular electrical stimulation; SSA: Standardized Swallowing Assessment; TMC: tongue motor cortex; PTT: pharyngeal motor cortex; MASA: Mann Assessment of Swallowing Ability; PSC: pharyngeal sensory cortex; ISC: inferior sensorimotor cortex; DOSS: Dysphagia Outcome and Severity Scale; FDS: Functional Dysphagia Scale; DSRS: Dysphagia Severity Rating Scale. 


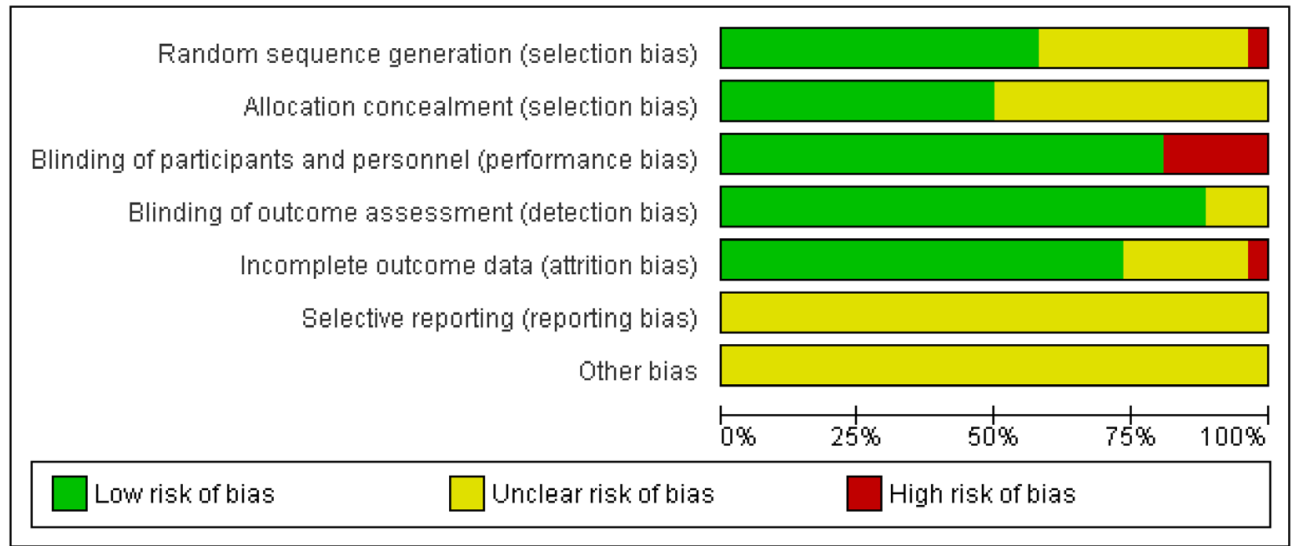

Figure 2. Risk of bias graph for all included studies.

considered potentially relevant. Two-hundred and seven duplicated studies were removed and 391 studies were excluded by screening the titles and abstracts. Forty studies went through fulltext assessment of eligibility and we excluded 14 studies for reasons including: no target intervention applied, not a randomized controlled trial, no target outcomes of relevance and nonrelevant study population. Twenty-six studies met the inclusion criteria and were included in systematic analysis and meta-analysis.

\section{Study Characteristics}

The included studies were all published between 2009 and 2020. The total number of patients included in this meta-analysis is 852. Among the included studies, 13 investigated treatment effects of rTMS with 335 patients, 7 studied tDCS effects with 201 patients, and 8 studied PES with 316 patients. Two studies investigated the effects of both rTMS ad PES. The mean age (SD) of patients was 66.0 (12.8) years. The time from stroke onset to intervention varied across studies, ranging from $30 \mathrm{~h}$ to 6 years (mean $[S D]=87.8[241.5]$ days). Table 1 summarizes the characteristics of all the included studies.

\section{Risk of Bias Assessment}

The risk of bias assessment results is presented in Figures 2 and 3. The majority of studies had a low risk of selection (except allocation concealment), performance, detection, and attribution bias. There was insufficient information to determine the risk of selective reporting and other risks so these two aspects were not further quantified.

\section{Outcome Measures}

The outcome measures used varied across studies. Penetration aspiration scale (PAS) (57) and Dysphagia Outcome and Severity Scale (DOSS) (58) were the most commonly used scales to reflect dysphagia severity. Other outcome measures include Mann Assessment of Swallowing Ability (MASA) (59) score, Standardized Swallowing Assessment (SSA)(60), Dysphagia Severity Rating Scale (DSRS) (28,61), Functional Dysphagia Scale (FDS) (62), Functional Oral Intake Scale (FOIS) (63), videofluoroscopic dysphagia scale (VDS) (64), dysphagia grade and timing of events in swallowing. Two studies used the proportion of patients ready for decannulation as outcome measure. Patients who were not decannulated were considered to have severe dysphagia and hence a failed outcome. Therefore, this outcome measure was considered relevant as the readiness for decannulation is closely related to their severity of dysphagia. No major adverse effects were reported across studies.

\section{Stimulation Duration}

Duration of stimulation varied across studies. Within a single session, the durations of rTMS ranged from 2 to $20 \mathrm{~min}$ and that of tDCS ranged from 20 to $30 \mathrm{~min}$, whereas the duration of PES was always $10 \mathrm{~min}$. The number of rTMS sessions ranged from one to ten days. Similarly, the number of tDCS sessions ranged from 4 to 20 days. PES had the most consistently reported number of sessions which were either one day or three days. As the stimulation duration is dependent on the chosen stimulation parameters, for example, intensity or frequency, to fulfill safety requirements, it was not possible to analyze this specific parameter as a separate factor in this meta-analysis.

\section{Meta-Analysis}

Overall Effects of Neurostimulation Compared to Control Treatments

Figure 4 presented the forest plot for pair-wise comparisons for rTMS, tDCS, and PES. The results showed that all three treatments yielded a moderate effect size compared with control treatments $(\mathrm{SMD}[95 \% \mathrm{Cl}]=0.69[0.50,0.89] ; p<0.001)$. The effect size of rTMS was the largest among the three interventions (SMD [95\% $\left.\mathrm{Cl}]=0.73[0.49,0.98] ; p<0.001 ; l^{2}=10 \%\right)$, followed by PES (SMD $\left.[95 \% \mathrm{Cl}]=0.68[0.22,1.14] ; p=0.004 ; l^{2}=65 \%\right)$ and tDCS (SMD $\left.[95 \% \mathrm{Cl}]=0.65[0.25,1.04] ; p=0.001 ; l^{2}=42 \%\right)$.

Given the high degree of heterogeneity for PES studies, sensitivity analysis was carried out. The heterogeneity was reduced $\left(I^{2}=34 \%\right)$ when the study by Bath and colleagues (56), which had high risk of bias for incomplete data was excluded. The resulting effect size for PES become the largest after adjustment (SMD $[95 \% \mathrm{Cl}]=0.83[0.43,1.42] ; p<0.001)$.

\section{Effects of Treatment Based on Follow-Up Period}

The follow-up period was categorized into three periods, including "early" which denoted follow-ups from immediate to two weeks post-treatment, "intermediate" which referred to follow-ups between three weeks and two months post-treatment, and "late" which referred to follow-ups from three months 


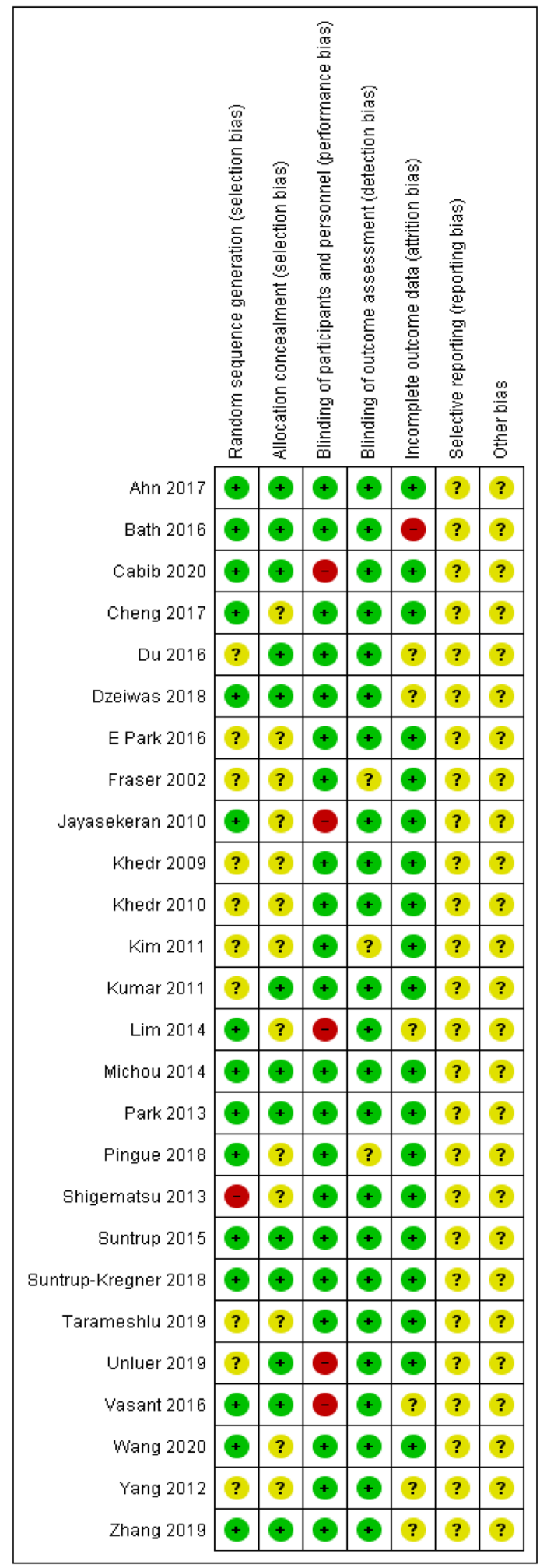

Figure 3. Risk of bias summary for individual studies.

onwards. The earliest follow-ups within the specified "early" period were analyzed, whereas the latest follow-ups within the specified "intermediate" and "late" period were analyzed. Figures 5-7 showed the meta-analysis results based on follow-up periods.
For early follow-up, all treatments showed comparable moderate effect sizes (rTMS: SMD [95\% Cl] $=0.69[0.46,0.93], p<0.001$; $l^{2}=0 \%$; PES: SMD $[95 \% \mathrm{Cl}]=0.68[0.22,1.14] ; p=0.004 ; l^{2}=65 \%$; tDCS: SMD $\left.[95 \% \mathrm{Cl}]=0.65[0.25,1.04] ; p=0.001 ; l^{2}=42 \%\right)$.

For intermediate follow-up, tDCS showed a larger pooled effect size $(\mathrm{SMD}[95 \% \mathrm{CI}]=1.74[0.67,2.80] ; p=0.001)$ than $\mathrm{rTMS}$ (SMD $\left.[95 \% \mathrm{Cl}]=1.02[0.45,1.59], p<0.001 ; l^{2}=65 \%\right)$. None of the PES studies reported intermediate follow-up data.

For late follow-up, none of the treatments showed significant effect sizes (rTMS: SMD [95\% Cl] $=0.78[-0.08,1.65], p=0.08$; $l^{2}=66 \%$; tDCS: SMD [95\% Cl] = $0.29[-0.78,1.35], p=0.60$; PES: $\left.\operatorname{SMD}[95 \% \mathrm{Cl}]=-0.04[-0.46,0.38], p=0.86 ; l^{2}=0 \%\right)$.

Effects of Treatment Based on Chronicity of Stroke

The chronicity of stroke was classified into acute (0-14 days), subacute (15-90 days), and chronic (beyond 90 days). The pooled effect size for acute stroke patients was large (SMD $[95 \% \mathrm{Cl}]=0.8$ $\left.[0.34,1.26], p<0.001 ; l^{2}=70 \% ;\right)$, whereas that for subacute and chronic stroke patients was moderate $(\mathrm{SMD}[95 \% \mathrm{Cl}]=0.75[0.46$, 1.04], $p<0.001 ; l^{2}=23 \%$ and SMD [95\% Cl] $=0.51[0.23,0.80]$, $p<0.001 ; l^{2}=0 \%$, respectively; Fig. 8). The heterogeneity for studies in acute stroke studies was much higher compared to more chronic studies.

Effects of Noninvasive Brain Stimulation Based on Stimulation Hemisphere

Subgroup analysis was conducted to compare the effects of stimulation hemisphere (ipsilesional vs. contralesional vs. bihemispheric) for NIBS studies (Fig. 9). All three stimulation sites showed significant effect size compared with controls (SMD $[95 \% \mathrm{Cl}]=0.71[0.51$, 0.92]; $p<0.001 ; l^{2}=18 \%$ ). Bihemispheric stimulation showed the strongest effect size $\left(\mathrm{SMD}[95 \% \mathrm{Cl}]=0.93[0.53,1.33] ; l^{2}=0 \%\right.$; $p<0.001)$, followed by contralesional (SMD $[95 \% \mathrm{Cl}]=0.73[0.46$, 0.99]; $\left.I^{2}=0 \% ; p<0.001\right)$ and ipsilesional (SMD $[95 \% \mathrm{Cl}]=0.62[0.14$, 1.10]; $l^{2}=55 \% ; p=0.01$ ) stimulation. It should be noted that the heterogeneity for trials reported as ipsilesional hemispheric stimulation was much higher than the other approaches.

Effects of rTMS Based on Stimulation Hemisphere and Frequency

Given the diversity of methodology for unilateral rTMS paradigms, further subgroup analyses were conducted (Fig. 10) with stimulated hemisphere and frequency being included. The subgroups included low-frequency ipsilesional hemisphere, high-frequency ipsilesional hemisphere, and high-frequency contralesional hemisphere.

The application of high-frequency rTMS over ipsilesional hemisphere showed the largest effect size (SMD $[95 \% \mathrm{Cl}]=0.83[0.14$, 1.52 ]; $\left.p=0.02 ; l^{2}=61 \%\right)$. The effect sizes for low-frequency over contralesional hemisphere $(\mathrm{SMD}[95 \% \mathrm{Cl}]=0.61[0.23,0.98]$; $\left.p=0.002 ; l^{2}=0 \%\right)$ and high-frequency over contralesional hemisphere $\left(\mathrm{SMD}[95 \% \mathrm{Cl}]=0.59[0.03,1.14] ; p=0.04 ; l^{2}=0 \%\right)$ were comparable. The high-frequency ipsilesional hemisphere approach showed considerably higher statistical heterogeneity $\left(I^{2}=61 \%\right)$ than other approaches, implying that the effects are less consistently reported across studies. Sensitivity analysis showed that for high-frequency ipsilesional subgroup, excluding the study by Khedr and colleagues (35) resulted in reduction of heterogeneity $\left(I^{2}=38 \%\right)$ and the overall effect became insignificant $(\mathrm{SMD}[95 \% \mathrm{Cl}]=0.57[-0.05,1.18] ; p=0.07)$. This study used $3 \mathrm{~Hz}$ rTMS, as opposed to 5 or $10 \mathrm{~Hz}$ rTMS used in other studies. 


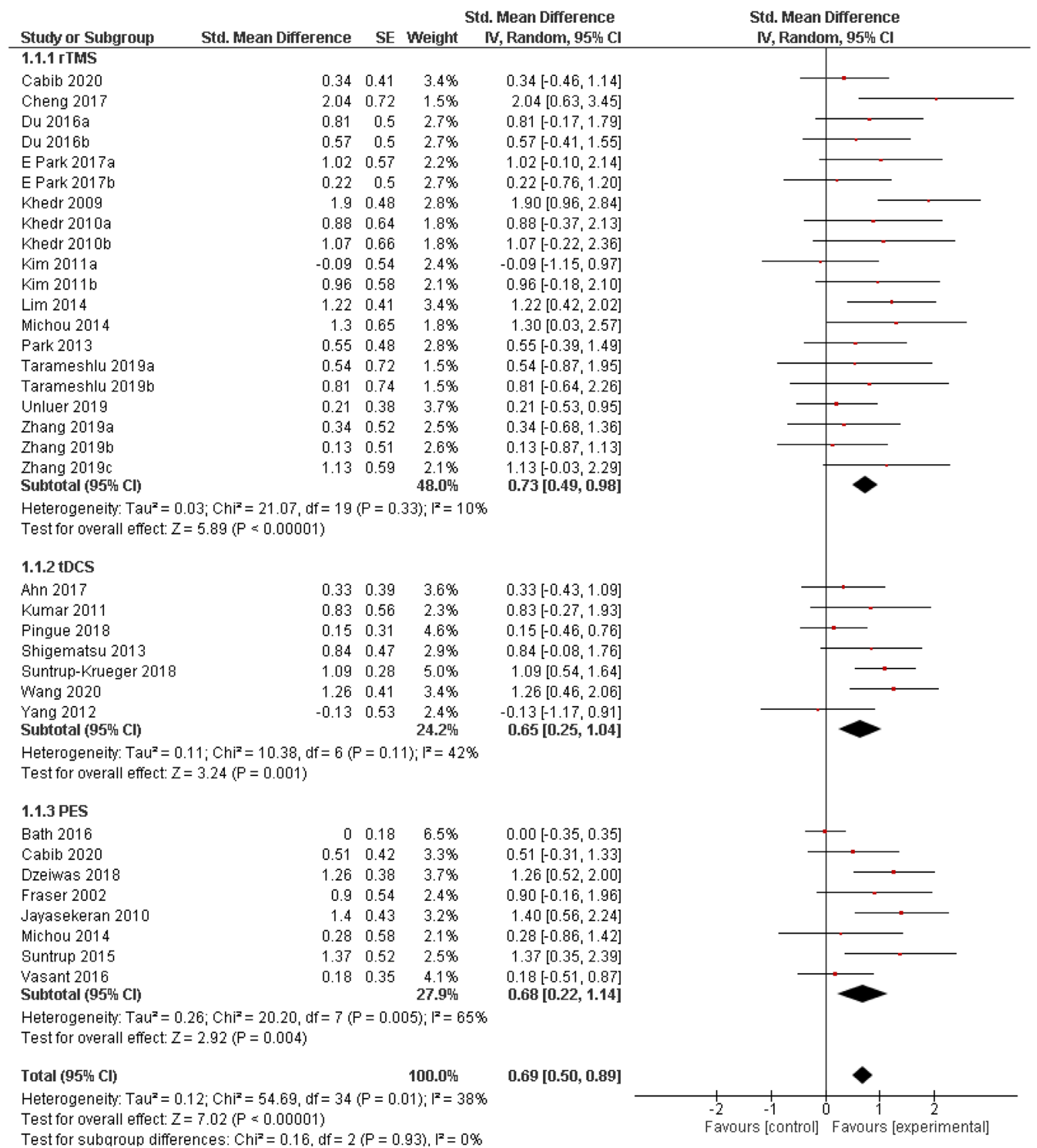

Figure 4. Forest plot showing overall effects of all three treatments compared to control treatment.

Effects of tDCS Based on Stimulated Hemisphere

All tDCS protocols used excitatory anodal tDCS, therefore, only the stimulated hemisphere was analyzed in the subgroup analysis (Fig. 11). Among the three stimulation sites (contralesional or ipsilesional hemisphere and bihemispheric stimulation), only the contralesional hemisphere approach showed a significant effect size $\left(\mathrm{SMD}[95 \% \mathrm{Cl}]=1.04[0.54,1.53] ; p<0.001 ; l^{2}=0 \%\right)$.

\section{DISCUSSION}

This systematic review and meta-analysis evaluated the effects of rTMS, tDCS, and PES on swallowing-related outcomes in patients with post-stroke dysphagia. We found that overall, these treatments are superior to conventional dysphagia treatments or sham stimulation. All interventions demonstrated moderate effect sizes, with rTMS showing an overall largest effect size, followed by PES and tDCS. No major adverse effects were reported across the studies analyzed. Our results suggested that, with a pooled sample size of 852 , these neurostimulation treatments are beneficial to patients with poststroke dysphagia. We analyzed different parameters of neurostimulation, which provided insights into and directions for future clinical practice.

\section{Neurostimulation as a Treatment for Poststroke Dysphagia}

Our results showed that both types of cortical neurostimulation (rTMS and tDCS) as well as peripheral stimulation (PES) are 


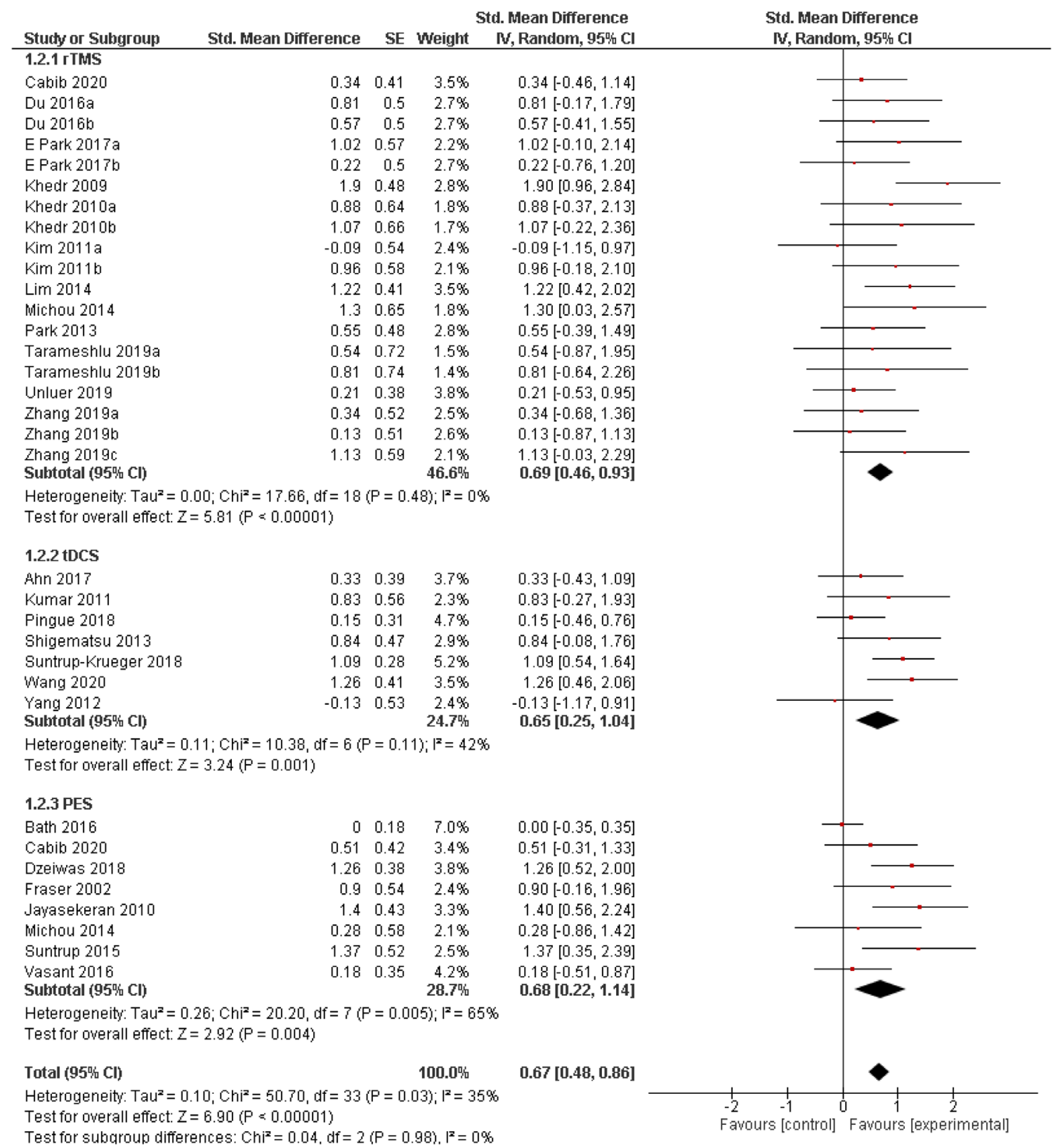

Figure 5. Forest plot showing "early" (up to two weeks) effects of all treatments.

effective in improving swallowing-related outcomes for stroke patients. The positive effects of rTMS and tDCS are in agreement with recent meta-analyses $(19,20,22)$. However, the meta-analysis by Chiang and colleagues (22) did not report positive effects for PES. A possible reason for the discrepancy is that more PES studies have been published since their meta-analysis was conducted and these were included in our review. Moreover, we included studies that used decannulation as an outcome measure $(26,27)$. This was considered a relevant outcome in our review because the severity of dysphagia is related to the readiness for decannulation and hence this could be used as a proxy for improved swallowing outcome. Inclusion of these studies allowed a better understanding on the effectiveness of PES for patients with more severe dysphagia. Taken together, the positive effects of these neurostimulation treatments suggested that dysphagia treatments targeting either the sensory or motor neural pathways of swallowing, depending on the patients' characteristics, can equivalently achieve beneficial outcomes. Some studies have suggested pairing cortical stimulation with peripheral stimulation, for example, paired-associated stimulation (PAS), and found positive results with chronic stroke patients with dysphagia $(39,65)$. However, PAS has only been applied in the short-term in two published studies and therefore was not included in this review.

\section{Effects of Neurostimulation Treatment Over Time}

We found that the effects of rTMS, tDCS and PES were most significant within the first two months of treatment compared to control treatment. This is in part due to the lack of studies reporting long term outcomes. In this review, only $20 \%(7 / 35)$ of the included trials reported outcomes of three months or beyond. The evidence for long-term effect is not sufficient to draw 


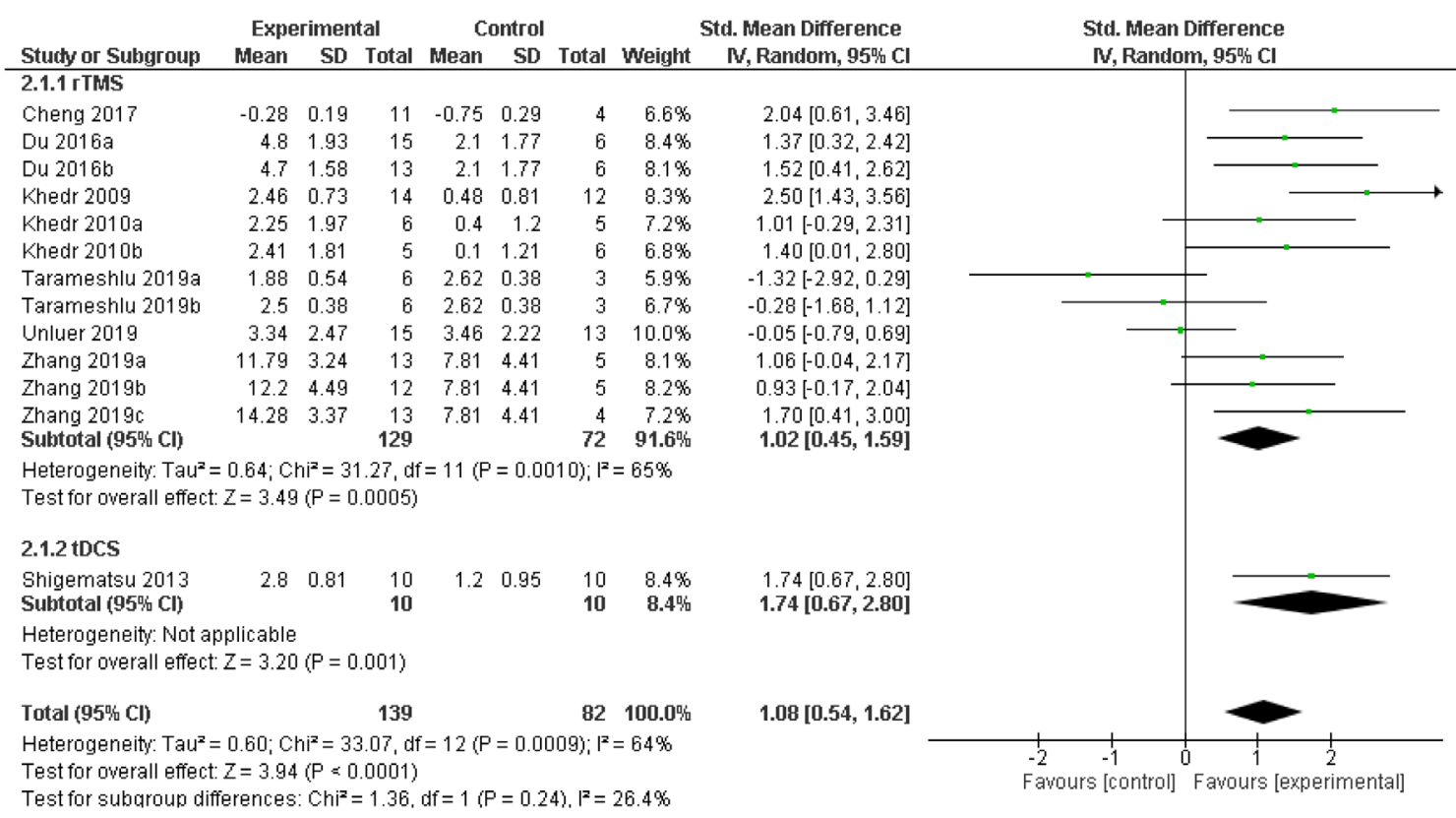

Figure 6. Forest plot showing "intermediate" (three weeks to two months) effects of all treatments.

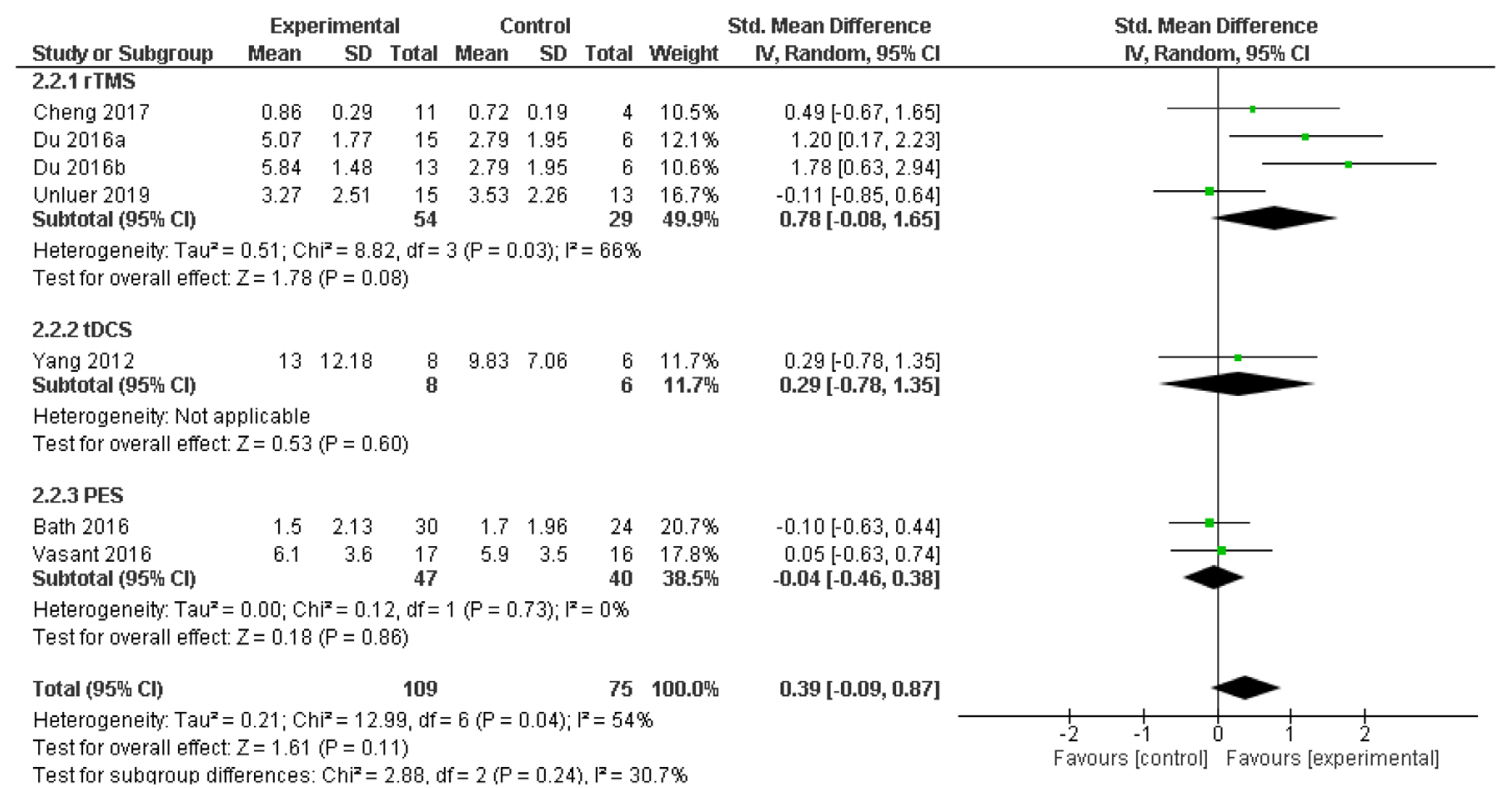

Figure 7. Forest plot showing "late" (three months or beyond) effects of all treatments.

definitive and meaningful conclusions. Notwithstanding, neurostimulation treatments may act as catalysts to enhance plasticity during the initial stages following treatment, accelerating recovery rather than reversing an incurable swallowing deficit, meaning that patients will still show some recovery over time even without stimulation based treatments. Studies have showed that functional recovery is the most significant within the first 90 days poststroke $(66,67)$. The course of treatment effects may follow this trajectory of recovery such that when the brain receives external plasticityinducing stimulation, positive functional changes are greatest within the initial three months and may demonstrate a ceiling effect after this period. Future studies may explore whether these treatments could have more sustained improvements in swallowing when given periodically or repeatedly as boosters, for example, every three months.

\section{Effects of Neurostimulation Treatment Based on Chronicity of Stroke}

Regarding the timing of treatment, we found that the effects were the strongest when applied during the first two weeks following stroke. This suggests that neurostimulation treatments may be more beneficial in accelerating functional (swallowing) recovery during the acute phase of stroke. However, this finding should be interpreted with cautions as there was large heterogeneity across studies with acute stroke patients. It is possible that 


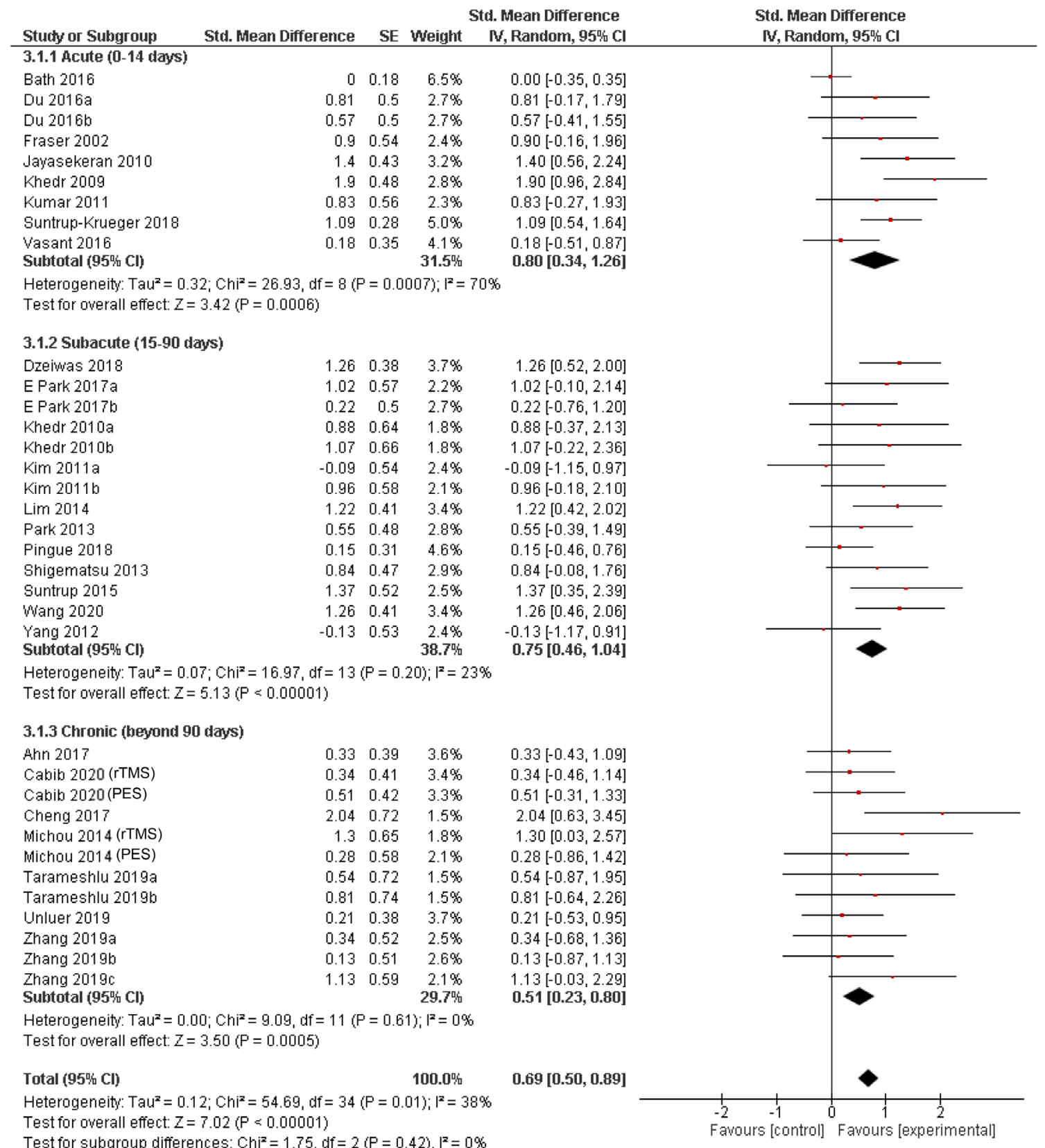

Figure 8. Forest plot showing effects of all treatments based on chronicity of stroke patients studied.

during this stage poststroke, the clinical condition of is relatively unstable, resulting in varied responsiveness toward these treatments.

\section{Effects of rTMS and tDCS Based on Stimulation Hemisphere}

We compared the treatment effects of rTMS and tDCS based on the hemisphere of stimulation. Our results showed that bihemispheric stimulation yielded the strongest effects among the three stimulation sites (ipsilesional, contraleisional, and bihemispheric). Given that the human swallowing system is bilaterally innervated, it seems reasonable to assume that bihemispheric stimulation would produce the greatest levels of benefit, presumably by promoting plasticity in both hemispheres, and driving significant functional recovery.
The findings from unilateral stimulation studies appeared to be more controversial. In general, the unilateral stimulation protocols were divided into two categories based on two different recovery models. The first model is the interhemispheric competition model, which assumes that the affected hemisphere would have reduced output and the unaffected hemisphere would exert excessive inhibition to the affected hemisphere. Based on this model, brain stimulation protocols are designed to restore the balance of such interhemispheric inhibition. Therefore, inhibitory stimulation such as low-frequency rTMS or cathodal tDCS could be applied to the contralesional hemisphere to suppress interhemispheric inhibition whereas excitatory stimulation such as high-frequency rTMS or anodal tDCS could be given to the ipsilesional hemisphere to increase the excitability of the affected hemisphere (68). The second model is the vicariation (or remote 


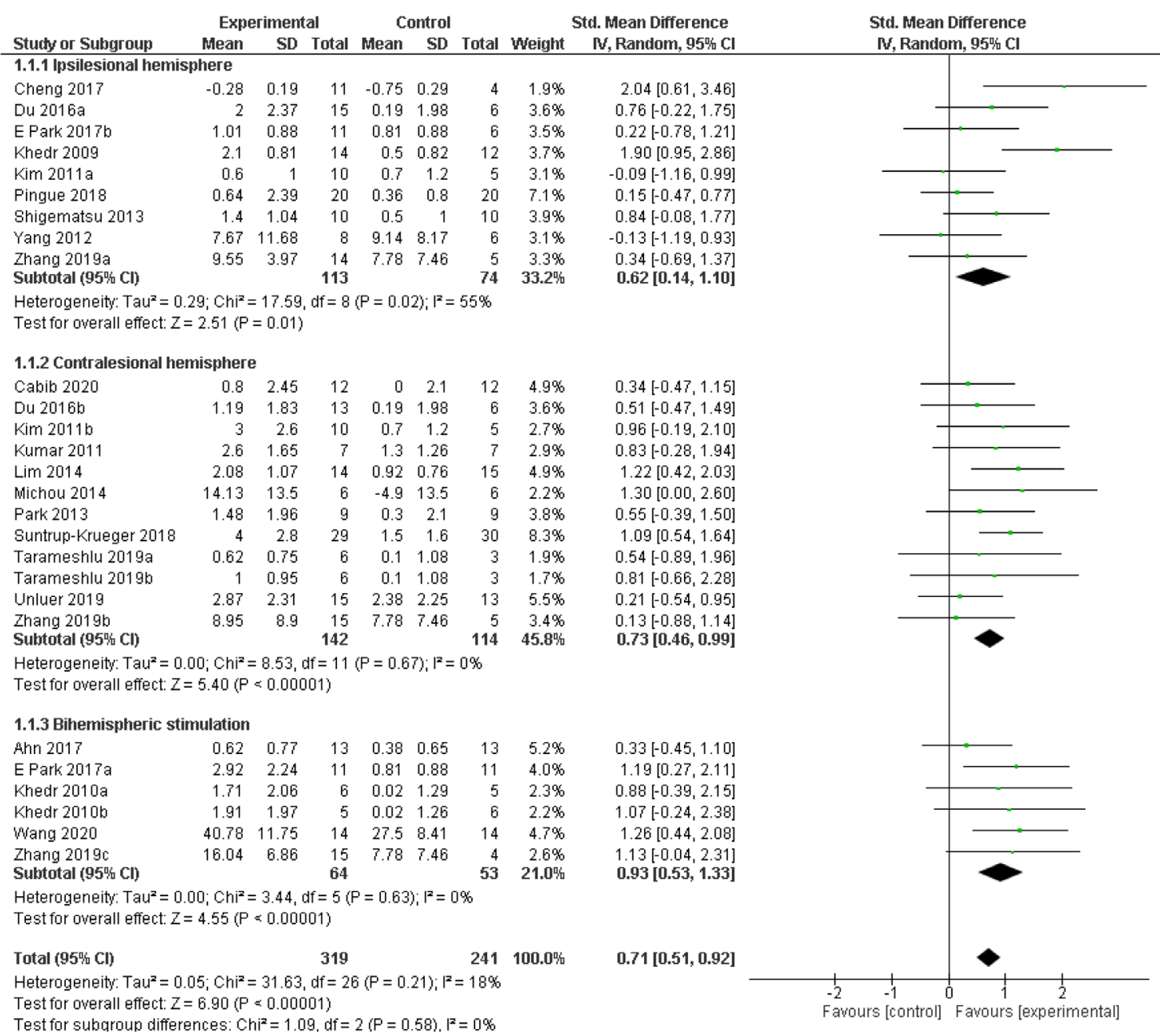

Figure 9. Forest plot showing subgroup analysis of effects of rTMS and tDCS based on stimulation hemisphere.

substrate compensation) model with supporting evidence from early brain mapping studies (14). It was found that recovery from poststroke dysphagia is associated with compensatory reorganization of the unaffected hemisphere (14). Therefore, based on this model, excitatory stimulation could be applied over the unaffected hemisphere to promote such reorganization.

Our results showed that all three unilateral stimulation approaches were effective in improving swallowing-related outcomes. Interestingly, ipsilesional excitatory stimulation protocol appeared to be most effective with rTMS, whereas contralesional excitatory stimulation protocol was more effective with tDCS. This discrepancy could be due to the differences in mechanisms of rTMS and tDCS such that the recruitment of neural networks differs following these treatments. More importantly, our hemispheric findings suggest that the recovery from poststroke dysphagia is more complex than either interhemispheric competition model or vicariation model alone. Recently, the bimodal balance-recovery model has been proposed to describe the course of neural plasticity changes following stroke (15). This model incorporates the concept of "structural reserve," which refers to the residual functional neural pathways that are capable for reorganization. The amount of structural reserve could determine which model, interhemispheric competition or vicariation, better predicts the functional outcomes. If the brain damage is extensive and structural reserve is low, then the input from the unaffected hemisphere would be critical to vicariate lost function. Hence, neurostimulation applied on the unaffected hemisphere may yield better functional outcomes in this scenario. On the contrary, if the structural reserve is high, then neurostimulation based on the interhemispheric inhibition model may be more appropriate. Therefore, we speculate that the efficacy of these approaches depends partly on the severity of brain damage of the patients recruited. However, patients with different stroke severities and lesion sites are often grouped together in these studies, making it difficult to isolate these relevant factors when analyzing the treatment effects. Future studies should explore protocols that are tailored to individual patient's prognosis based on their stroke characteristics.

\section{LIMITATIONS}

There are several limitations for this review. First, the outcome measures and treatment protocols were highly heterogeneous, 


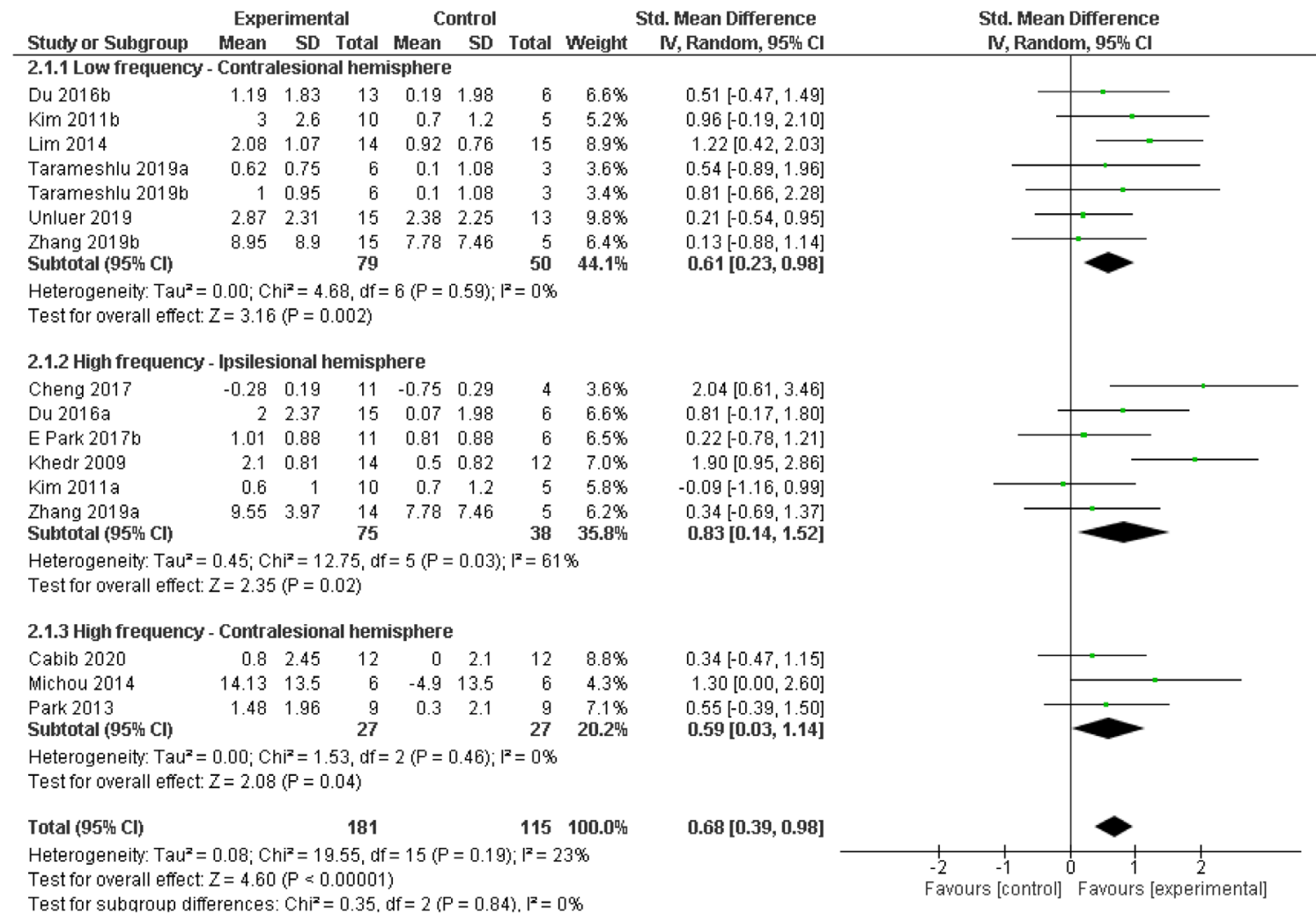

Figure 10. Forest plot showing subgroup analysis on the effects of rTMS based on stimulation hemisphere and frequency.

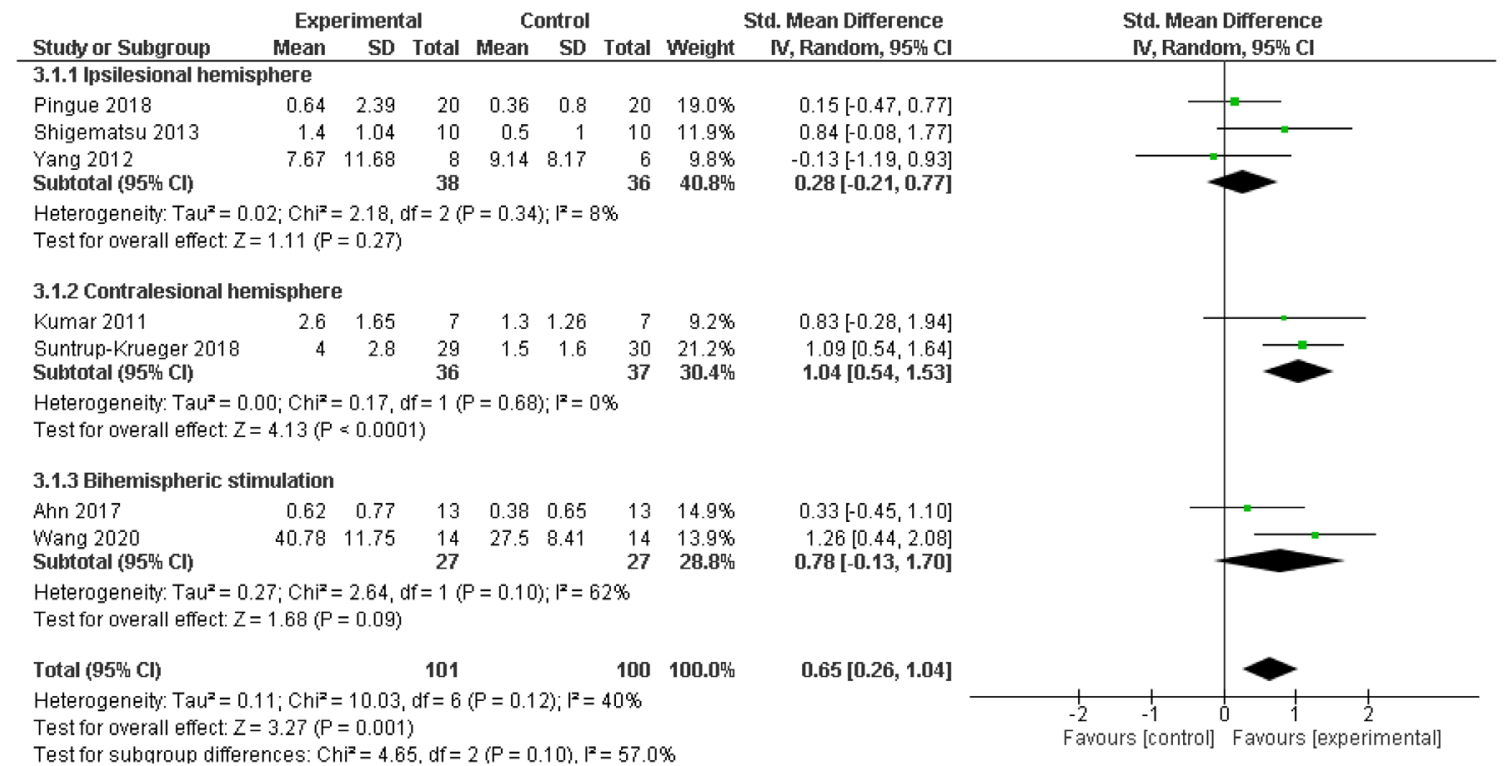

Figure 11. Forest plot showing subgroup analysis on effects of tDCS based on stimulation hemisphere.

making comparisons across studies and the estimation of true treatment effects challenging. Moreover, the heterogeneity of patient characteristics precludes a generalized conclusion on the effectiveness of the treatments. Therefore, the context of the reported findings and mixed methodologies applied should be taken into account when interpreting our results.

\section{CONCLUSIONS}

In conclusion, our systematic review found that rTMS, tDCS, and PES have beneficial effects on swallowing-related outcomes for stroke patients with dysphagia compared to conventional dysphagia treatment or sham stimulation. Subgroup analysis showed 
that the effects of these treatments were the strongest when applied during the first two weeks following stroke and within the first two months of application. Moreover, bihemispheric stimulation protocols for noninvasive brain stimulation appeared to be most effective in improving swallowing. No major adverse effects were reported across the reported studies included in our review. Future studies should, we propose, explore neurostimulation protocols that are tailored to individual patient's stroke characteristics and prognosis.

\section{Authorship Statements}

All authors contributed substantially to conception and design of the review, acquisition, analysis and interpretation of data, drafting the article and reviewing it critically for important intellectual content. All authors approved the final version of the article.

\section{How to Cite this Article:}

Cheng I., Sasegbon A., Hamdy S. 2021. Effects of Neurostimulation on Poststroke Dysphagia: A Synthesis of Current Evidence From Randomized Controlled Trials. Neuromodulation 2021; 24: 1388-1401

\section{REFERENCES}

1. Martino R, Foley N, Bhogal S, Diamant N, Speechley M, Teasell R. Dysphagia after stroke: incidence, diagnosis, and pulmonary complications. Stroke 2005;36: 2756-2763.

2. Arnold M, Liesirova $\mathrm{K}$, Broeg-Morvay $\mathrm{A}$ et al. Dysphagia in acute stroke: incidence, burden and impact on clinical outcome. PLoS One. 2016;11:e0148424.

3. Smithard DG, O'Neill PA, Parks C, Morris J. Complications and outcome after acute stroke. Does dysphagia matter? Stroke 1996;27:1200-1204.

4. Ekberg O, Hamdy S, Woisard V, Wuttge-Hannig A, Ortega P. Social and psychological burden of dysphagia: its impact on diagnosis and treatment. Dysphagia 2002;17:139-146.

5. Smithard DG, O'Neill PA, England RE et al. The natural history of dysphagia following a stroke. Dysphagia Fall 1997;12:188-193.

6. Muehlemann $N$, Jouaneton $B$, de Leotoing $L$ et al. Hospital costs impact of post ischemic stroke dysphagia: Database analyses of hospital discharges in France and Switzerland. PLoS One 2019;14:e0210313.

7. Bonilha HS, Simpson AN, Ellis C, Mauldin P, Martin-Harris B, Simpson K. The oneyear attributable cost of post-stroke dysphagia. Dysphagia 2014;29:545-552.

8. Bath PM, Lee HS, Everton LF. Swallowing therapy for dysphagia in acute and subacute stroke. Cochrane Database Syst Rev 2018;10:CD000323.

9. Miller AJ. Neurophysiological basis of swallowing. Dysphagia 1986;1:91.

10. Martin RE, Kemppainen P, Masuda Y, Yao D, Murray GM, Sessle BJ. Features of cortically evoked swallowing in the awake primate (Macaca fascicularis). J Neurophysiol 1999;82:1529-1541.

11. Sumi T. Some properties of cortically-evoked swallowing and chewing in rabbits. Brain Res 1969;15:107-120.

12. Hamdy S, Aziz Q, Rothwell JC et al. The cortical topography of human swallowing musculature in health and disease. Nat Med 1996;2:1217-1224.

13. Hamdy S, Aziz Q, Rothwell JC et al. Explaining oropharyngeal dysphagia after unilateral hemispheric stroke. Lancet 1997;350:686-692.

14. Hamdy S, Aziz Q, Rothwell JC et al. Recovery of swallowing after dysphagic stroke relates to functional reorganization in the intact motor cortex. Gastroenterology 1998;115:1104-1112.

15. Di Pino G, Pellegrino G, Assenza G et al. Modulation of brain plasticity in stroke: a novel model for neurorehabilitation. Nat Rev Neurol 2014;10:597-608.

16. Fregni F, Pascual-Leone A. Technology insight: noninvasive brain stimulation in neurology-perspectives on the therapeutic potential of rTMS and tDCS. Nat Clin Pract Neurol 2007:3:383-393.

17. Hallett M. Transcranial magnetic stimulation and the human brain. Nature 2000 406:147-150

18. Nitsche MA, Paulus W. Excitability changes induced in the human motor cortex by weak transcranial direct current stimulation. J Physiol 2000;527:633-639.
19. Marchina S, Pisegna JM, Massaro JM et al. Transcranial direct current stimulation for post-stroke dysphagia: a systematic review and meta-analysis of randomized controlled trials. J Neurol 2020;268:1-12.

20. Yang SN, Pyun SB, Kim HJ, Ahn HS, Rhyu BJ. Effectiveness of non-invasive brain stimulation in dysphagia subsequent to stroke: a systemic review and meta-analysis. Dysphagia 2015;30:383-391.

21. Liao X, Xing GQ, Guo ZW et al. Repetitive transcranial magnetic stimulation as an alternative therapy for dysphagia after stroke: a systematic review and metaanalysis. Clin Rehabil 2017;31:289-298.

22. Chiang CF, Lin MT, Hsiao MY, Yeh YC, Liang YC, Wang TG. Comparative efficacy of noninvasive neurostimulation therapies for acute and subacute poststroke dysphagia: a systematic review and network meta-analysis. Arch Phys Med Rehabil 2019;100:739-750. e734.

23. Pisegna JM, Kaneoka A, Pearson WG, Kumar S, Langmore SE. Effects of noninvasive brain stimulation on post-stroke dysphagia: a systematic review and meta-analysis of randomized controlled trials. Clin Neurophysiol 2016;127: 956-968.

24. Hamdy S, Rothwell JC, Aziz Q, Singh KD, Thompson DG. Long-term reorganization of human motor cortex driven by short-term sensory stimulation. Nat Neurosci 1998;1:64-68.

25. Fraser $C$, Power $M$, Hamdy $S$ et al. Driving plasticity in human adult motor cortex is associated with improved motor function after brain injury. Neuron 2002;34 $831-840$

26. Dziewas R, Stellato R, van der Tweel I et al. Pharyngeal electrical stimulation for early decannulation in tracheotomised patients with neurogenic dysphagia after stroke (PHAST-TRAC): a prospective, single-blinded, randomised trial. Lancet $\mathrm{Neu}$ rol 2018:17:849-859.

27. Suntrup S, Marian T, Schroder JB et al. Electrical pharyngeal stimulation for dysphagia treatment in tracheotomized stroke patients: a randomized controlled trial. Intensive Care Med 2015;41:1629-1637.

28. Jayasekeran V, Singh S, Tyrrell $P$ et al. Adjunctive functional pharyngeal electrical stimulation reverses swallowing disability after brain lesions. Gastroenterology 2010;138:1737-1746.

29. Sasegbon A, Cheng I, Zhang M, Hamdy S. Advances in the use of neuromodulation for neurogenic dysphagia: mechanisms and therapeutic application of pharyngeal electrical stimulation, transcranial magnetic stimulation, and transcranial direct current stimulation. Am J Speech Lang Pathol 2020;29: 1044-1064.

30. Lajeunesse MJ, Koricheva J, Gurevitch J, Mengersen K. Recovering missing or partial data from studies: a survey of conversions and imputations for meta-analysis. Handbook of meta-analysis in ecology and evolution. Princeton, NJ: Princeton University Press 2013:195-206.

31. Higgins JPT, Thomas J, Chandler J, Cumpston M, Li T, Page MJ, Welch VA (editors). Cochrane Handbook for Systematic Reviews of Interventions version 6.1 (updated September 2020). Cochrane, 2020. Available from www.training. cochrane.org/handbook.

32. Luo D, Wan X, Liu J, Tong T. Optimally estimating the sample mean from the sample size, median, mid-range, and/or mid-quartile range. Stat Methods Med Res 2018;27:1785-1805.

33. Wan X, Wang WQ, Liu JM, Tong TJ. Estimating the sample mean and standard deviation from the sample size, median, range and/or interquartile range. $B M C$ Med Res Methodol 2014;19:14.

34. Cohen J. Statistical power analysis for the behavioral sciences. London, UK: Academic Press, 2013

35. Khedr EM, Abo-Elfetoh N, Rothwell JC. Treatment of post-stroke dysphagia with repetitive transcranial magnetic stimulation. Acta Neurol Scand 2009;119:155-161.

36. Khedr EM, Abo-Elfetoh N. Therapeutic role of rTMS on recovery of dysphagia in patients with lateral medullary syndrome and brainstem infarction. $J$ Neurol Neurosurg Psychiatry 2010;81:495-499.

37. Kim L, Chun MH, Kim BR, Lee SJ. Effect of repetitive transcranial magnetic stimulation on patients with brain injury and dysphagia. Ann Rehabil Med 2011;35:765-771.

38. Park JW, Oh JC, Lee JW, Yeo JS, Ryu KH. The effect of $5 \mathrm{~Hz}$ high-frequency rTMS over contralesional pharyngeal motor cortex in post-stroke oropharyngeal dysphagia: A randomized controlled study. Neurogastroenterol Motil 2013;25:324-e250.

39. Michou E, Mistry S, Jefferson S, Tyrrell P, Hamdy S. Characterizing the mechanisms of central and peripheral forms of neurostimulation in chronic dysphagic stroke patients. Brain Stimul 2014;7:66-73.

40. Lim KB, Lee HJ, Yoo J, Kwon YG. Effect of low-frequency rTMS and NMES on subacute unilateral hemispheric stroke with dysphagia. Ann Rehabil Med 2014;38: 592-602.

41. Du J, Yang F, Liu L et al. Repetitive transcranial magnetic stimulation for rehabilitation of poststroke dysphagia: a randomized, double-blind clinical trial. Clin Neurophysiol 2016;127:1907-1913.

42. Park E, Kim MS, Chang WH et al. Effects of bilateral repetitive transcranial magnetic stimulation on post-stroke dysphagia. Brain Stimul 2017;10:75-82.

43. Cheng IKY, Chan KMK, Wong CS et al. Neuronavigated high-frequency repetitive transcranial magnetic stimulation for chronic post-stroke dysphagia: a randomized controlled study. J Rehabil Med 2017:49:475-481.

44. Tarameshlu M, Ansari NN, Ghelichi L, Jalaei S. The effect of repetitive transcranial magnetic stimulation combined with traditional dysphagia therapy on poststroke dysphagia: A pilot double-blinded randomized-controlled trial. Int $J$ Rehabil Res 2019:42:133-138.

45. Unluer NO, Temucin CM, Demir N, Serel Arslan S, Karaduman AA. Effects of lowfrequency repetitive transcranial magnetic stimulation on swallowing function and quality of life of post-stroke patients. Dysphagia 2019;34:360-371. 
46. Zhang $C$, Zheng X, Lu R, Yun W, Yun $H$, Zhou X. Repetitive transcranial magnetic stimulation in combination with neuromuscular electrical stimulation for treatment of post-stroke dysphagia. J Int Med Res 2019;47:662-672.

47. Cabib C, Nascimento W, Rofes L et al. Short-term neurophysiological effects of sensory pathway neurorehabilitation strategies on chronic poststroke oropharyngeal dysphagia. Neurogastroenterol Motil 2020;32:e13887.

48. Kumar $\mathrm{S}$, Wagner $\mathrm{CW}$, Frayne $\mathrm{C}$ et al. Noninvasive brain stimulation may improve stroke-related dysphagia: A pilot study. Stroke 2011;42:1035-1040.

49. Yang EJ, Baek SR, Shin J et al. Effects of transcranial direct current stimulation (tDCS) on post-stroke dysphagia. Restor Neurol Neurosci 2012;30:303-311.

50. Shigematsu T, Fujishima I, Ohno K. Transcranial direct current stimulation improves swallowing function in stroke patients. Neurorehabil Neural Repair 2013;27:363-369.

51. Ahn YH, Sohn HJ, Park JS et al. Effect of bihemispheric anodal transcranial direct current stimulation for dysphagia in chronic stroke patients: a randomized clinical trial. J Rehabil Med 2017;49:30-35.

52. Suntrup-Krueger $S$, Ringmaier $C$, Muhle $P$ et al. Randomized trial of transcranial direct current stimulation for poststroke dysphagia. Ann Neurol 2018;83:328-340.

53. Pingue V, Priori A, Malovini A, Pistarini C. Dual transcranial direct current stimulation for Poststroke dysphagia: a randomized controlled trial. Neurorehabil Neural Repair 2018;32:635-644.

54. Wang ZY, Chen JM, Lin ZK, Ni GX. Transcranial direct current stimulation improves the swallowing function in patients with cricopharyngeal muscle dysfunction following a brainstem stroke. Neurol Sci 2020;41:569-574.

55. Vasant $\mathrm{DH}$, Michou $\mathrm{E}, \mathrm{O}^{\prime}$ Leary $\mathrm{N}$ et al. Pharyngeal electrical stimulation in dysphagia poststroke: a prospective, randomized single-blinded interventional study. Neurorehabil Neural Repair 2016;30:866-875.

56. Bath PM, Scutt P, Love J et al. Pharyngeal electrical stimulation for treatment of dysphagia in subacute stroke: a randomized controlled trial. Stroke 2016;47:1562-1570.
57. Rosenbek JC, Robbins JA, Roecker EB, Coyle JL, Wood JL. A penetrationaspiration scale. Dysphagia 1996;11:93-98.

58. O'Neil KH, Purdy M, Falk J, Gallo L. The dysphagia outcome and severity scale. Dysphagia 1999;14:139-145.

59. Mann G. MASA: the Mann assessment of swallowing ability. Vol 1. Boston, MA Cengage Learning, 2002

60. Perry L. Screening swallowing function of patients with acute stroke. Part two: detailed evaluation of the tool used by nurses. J Clin Nurs 2001;10:474-481.

61. Everton LF, Benfield JK, Hedstrom A et al. Psychometric assessment and validation of the dysphagia severity rating scale in stroke patients. Sci Rep 2020;10: 7268.

62. Han TR, Paik NJ, Park JW. Quantifying swallowing function after stroke: a functional dysphagia scale based on videofluoroscopic studies. Arch Phys Med Rehabil 2001;82:677-682.

63. Crary MA, Mann GD, Groher ME. Initial psychometric assessment of a functional oral intake scale for dysphagia in stroke patients. Arch Phys Med Rehabil 2005;86: 1516-1520.

64. Han TR, Paik NJ, Park JW, Kwon BS. The prediction of persistent dysphagia beyond six months after stroke. Dysphagia 2008;23:59-64.

65. Michou E, Mistry S, Jefferson S, Singh S, Rothwell J, Hamdy S. Targeting unlesioned pharyngeal motor cortex improves swallowing in healthy individuals and after dysphagic stroke. Gastroenterology 2012;142:29-38.

66. Murphy $\mathrm{TH}$, Corbett D. Plasticity during stroke recovery: from synapse to behaviour. Nat Rev Neurosci 2009;10:861-872.

67. Maulden SA, Gassaway J, Horn SD, Smout RJ, DeJong G. Timing of initiation of rehabilitation after stroke. Arch Phys Med Rehabil 2005;86:S34-S40.

68. Alia C, Spalletti C, Lai S et al. Neuroplastic changes following brain ischemia and their contribution to stroke recovery: novel approaches in neurorehabilitation. Front Cell Neurosci 2017;16:11. 